\title{
Strength of Joining Seams in Glass Welded by Ultra-fast Lasers Depending on Focus Height
}

\author{
Kristian CVECEK ${ }^{* 1}$, Isamu MIYAMOTO ${ }^{* 1,2,4}$, Johannes STRAUSS ${ }^{* 1}$, Viet BUI ${ }^{* 1}$, Sonja SCHARFENBERG ${ }^{* 1}$, Thomas \\ FRICK $^{* 1,4}$ and Michael SCHMIDT ${ }^{* 1,3,4}$ \\ ${ }^{* 1}$ Bayerisches Laserzentrum GmbH, Konrad-Zuse-Str. 2-6, 91052 Erlangen, Germany \\ E-mail: k.cvecek@blz.org \\ *2 Osaka University, 1-12 Yamada-Oka, Suita, Osaka 565-0871, Japan \\ ${ }^{* 3}$ Chair of Photonic Technologies, Friedrich-Alexander University Erlangen-Nürnberg, Germany \\ ${ }^{* 4}$ SAOT-Erlangen Graduate School in Advanced Optical Technologies, Friedrich-Alexander Uni- \\ versity Erlangen-Nürnberg, Germany
}

\begin{abstract}
Glass welding by ultra short laser pulses allows joining without the need of an absorber or a preand post-heating process. However, in order to achieve high joining strength the processing parameters such as feed speed, repetition rate, laser power and focal height must be chosen correctly. We show in this work that albeit the shear force of the joining seam depends on the displacement between the focal position and the joining interface the focal height can be chosen in a relatively wide range to provide welding seams with good quality. Furthermore, two different ways are examined in order how to measure the shear strength of a welding seam inside optically contacted area.
\end{abstract}

DOI:10.2961/jlmn.2012.01.0013

Keywords: Laser materials processing, glass and other amorphous materials, glass welding, ultrafast lasers, welding

\section{Introduction}

Glass is a very popular material due to its excellent physical and chemical properties. Its application can be found throughout optics, telecommunications, electronics, MEMS, biomedicine up to construction industry as well as design. However, in order to be of use for these applications an assembly or joining of several glass parts is required. In this case, the physical properties which make the glass excel in its application make it necessary to use elaborate technology to join it.

Nonetheless, several established technologies do already exist for this task. However, each glass joining technology so far has specific properties that make it useful only within a limited application range. In general, joining methods that use additional material such as in mechanical mounting [1], gluing [2] or soldering suffer often from a mismatch of thermal expansion coefficients between glass and mount material, glue or solder. This limits e.g. the temperature range in which such composites can be used. Moreover UV-curable glues are often hazardous to health and undergo a shrinkage process that leads to inherent stresses in the joining area. In comparison to these joining methods where additives are used also methods where no additives are necessary can be applied for glass joining. Such are e.g. optical contacting [3], thermal diffusion bonding [4] or $\mathrm{CO}_{2}$-laser welding [5]. Here again the methods are not suited for all purposes: optical contacting is susceptible to impact loads [6], thermal diffusion bonding requires long processing times and $\mathrm{CO}_{2}$-laser welding cannot be used on glass types other than fused silica without pre- and post-heating.

By contrast, in the last few years a promising approach for glass joining has been described, where no additives are necessary while the joining area achieves mechanical properties such as shear strength and bonding energy that are comparable with the base material $[7,8]$. This joining method uses ultra-short laser pulses that are focused strongly into the transparent glass material, whereby a plasma is generated inside the focal spot. The plasma heats and melts the adjacent material. The dimensions of the molten zone can be set by welding parameters such as laser power or feed rate. The parameters must be chosen such that the resulting welding seam is crack free, since an external mechanical load can cause nearly singular stress fields at the tips of an already existing crack [9], thus enabling further crack propagation.

However, feed rate and laser power are not the only parameters that influence the crack generation mechanism. A further criterion is the sample preparation method. In [7] has been shown, that optical contacting helps strongly to provide welding seams with high seam strength.

Closely related to the issue of defect-free welding is the displacement of the focal spot versus the joining surface of the optical contact. The molten zone of a welding seam is typically tear drop shaped with the tip located at the focal spot position and the "round" region of the tear located above the focal spot [10]. This shape originates in absorption processes inside the plasma which cause the plasma region and thus the molten zone to grow towards the laser source. Observation of welding seams under crossed polarizers shows qualitatively that the stress induced birefringence depends on the displacement of the focal spot to the joining surface [10]. Moreover, [10] shows that for focal displacements too close to the joining surface defects can occur inside the welding seam which might compromise the strength of the joint. In the present work the shear force 
and shear strength depending on the focal displacement from the joining surface are investigated. Since the investigated glass samples were prebonded by optical contacting in order to suppress defect generation during welding the contribution of the prebonded area must be taken into account. Therefore, in this work two different methods are presented and evaluated in order to find the best solution to determine the shear strength of the welding seam only.

\section{Experiment}

Prior to welding the glass samples were prepared by optical contacting of glass slides. This was done in order to avoid crack generation during welding [7]. As glass borosilicate D263 T with a thickness of $1 \mathrm{~mm}$ has been used. Since the surface quality of these plates was not intended for optical contacting, it was not possible to achieve optical contact across the whole area of the glass plates. Nonetheless the achieved area was still sufficiently large to accommodate the welding seam. The samples were prebonded in such a way that parts of the plates extended to the sides as shown in the inset of Fig. 1.

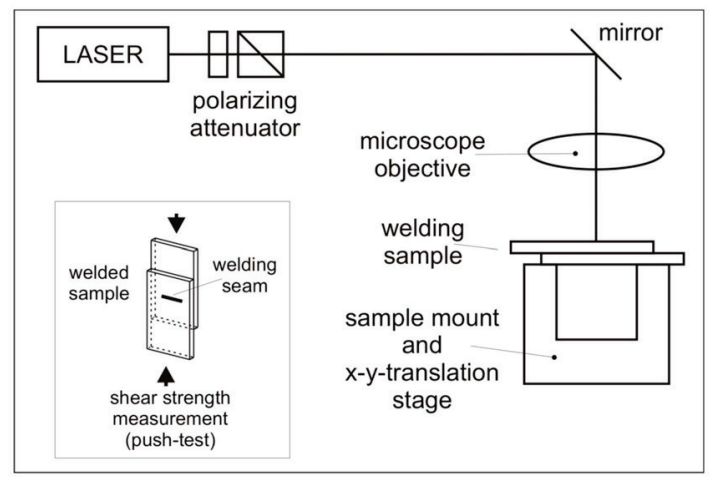

Fig. 1 Schematics of the experimental setup used for glass welding. The inset shows the shear strength measurement.

The experimental welding setup is depicted in Fig. 1. The laser used for welding was Fuego from TimeBandwidth Company with a FWHM-pulse duration of approx. $10 \mathrm{ps}$ at $1064 \mathrm{~nm}$. The repetition rate was set to $1 \mathrm{MHz}$. The average laser power was set electronically by an internal polarizing attenuator inside the laser system. The beam is then focused onto the sample by an IR microscope objective with a numerical aperture of 0.55 , a magnification of 50x and a working distance of $6 \mathrm{~mm}$. The objective was mounted on an automated z-positioning stage. In combination with the objective a camera was used in a confocal setup in order to observe the focus of the laser beam on the sample. The pre-bonded glass samples were mounted on an $\mathrm{x}$-y-translation stage. The average power used for welding was $2 \mathrm{~W}$ and the feed rate was set to $20 \mathrm{~mm} / \mathrm{s}$.

The samples were aligned first horizontally by measuring the distance from the microscope objective to the upper surface of the sample for several x-y-positions. From the measured heights angles have been calculated by which the sample must be tilted in order to have the upper surface of the samples in horizontal position. In a second step the lower surface of the upper glass plate was found with the same confocal setup. As the optical contact provides no reflections this was performed on a portion of the upper glass plate of the sample that extended over the lower glass plate. The detected position of the rear surface of the upper plate has been used as the reference height from which the focal spot is displaced. The displacement was set geometrically to $0 \mu \mathrm{m},-10 \mu \mathrm{m},-20 \mu \mathrm{m},-30 \mu \mathrm{m}$ and $-40 \mu \mathrm{m}$. Please notice that due to the geometrical displacement the true distance of the focal spot to the joining surface is exaggerated by the refractive index of glass, in this case approximately 1.52 [12]. The negative sign shall indicate that the focal spot was displaced below the reference height. The repeatability of the positioning stage was smaller than $4 \mu \mathrm{m}$.

Two different shear test experiments have been carried out in order to evaluate the dependency of the shear force of the welding seam on the focal displacement as well as to characterize the feasibility of the shear tests. The reason for this approach was the prebonding of the samples. The prebonding holds the glass plates by Van der Waals forces. Due to the much larger prebonding area in comparison with the welding seam area the shear forces exhibited by the prebonding are larger than the shear force necessary for the welding seam. Thus the optical contact must be taken into account if the shear force or strength of the welding seam is to be measured.

This can be done in a straightforward way, where the shear force is measured for prebonded as well as prebonded and welded samples and afterwards the averaged shear forces for each group are subtracted from each other and divided by the area of the welding seam [7]. In the present case the procedure was further refined so that prior to the shear test the prebonded area was measured. The measurement was performed by scanning the glass samples with a digital flatbed scanner while the prebonded area was deduced from the scanned image using commercial image processing software. Knowing the prebonded area of the welded samples allows to choose prebonded samples with a similar prebonding area to calculate the shear strength. The length of the welding in this experiment was set to $9 \mathrm{~mm}$ and for each focal displacement 3 samples were used.

The approach for other shear test experiment was again to reduce the influence of the prebonded area on the welding seam. The basis for this was found in preliminary experiments where it was observed that prebonded glass samples (especially those with lower surface quality) that are not welded tend to separate completely after some time if put into water or alcohol. By contrast welded samples did not separate. Therefore, welded samples were prepared prior to the measurements by immersing them for $15 \mathrm{~h}$ in a $98.4 \%$ ethanol solution. In this case ethanol was chosen since its surface tension is significantly lower than the surface tension of water while its viscosity is only marginally higher $[13,14]$. In this way the ethanol solution should permeate the sample more thoroughly while the lubrication of the samples is similar to water. In this experiment the welding seam length was $8 \mathrm{~mm}$ and for each focal displacement 4 glass samples were welded. The shear strength was calculated directly by dividing the measured force by the welding seam area.

In both experiments the width of the broken welding seam was measured by an optical microscope along the seam at several positions. The welding seam area was calculated by multiplying the average value of the seams width by the length of the welding seam. 


\section{Results}

Fig. 2 shows the measured shear forces for prebonded as well as welded samples. The force measured for welded samples is marked according to the focal displacement with respect to the joining surface. It can be clearly seen that although the shear force of just prebonded samples fluctuates the shear force for the displacements of $-20 \mu \mathrm{m}$ and $-30 \mu \mathrm{m}$ lie above the majority of prebonded samples. By contrast the shear forces of the displacements of $0 \mu \mathrm{m}$, $-10 \mu \mathrm{m}$ and $-40 \mu \mathrm{m}$ lie somewhat lower than the majority of shear forces measured for prebonded samples.

It is obvious that the scattering of the measured shear force is very large so that only very approximate values for the shear strength of a welding seam can be calculated.

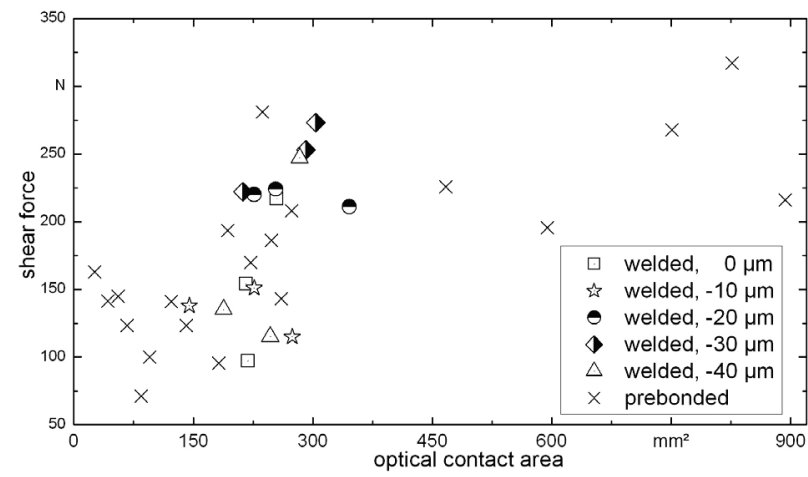

Fig. 2 Shear forces measured in dependence of the optical contact area for prebonded samples as well as prebonded and welded samples. The focal spot was displaced below the joining surface.

This fact is even more enhanced in fig. 3 which shows that only focal displacements of $-20 \mu \mathrm{m}$ and $-30 \mu \mathrm{m}$ lie above the average value for prebonded samples. For other focal displacements a calculation of shear strength as described in section 2 is not possible. Nonetheless, the shear strength was calculated using shear forces in fig. 3 and the measured welding seam areas. Table 1 shows the shear strength along with the values used for calculation. The measurement uncertainty was determined by the Gaussian law of error propagation.

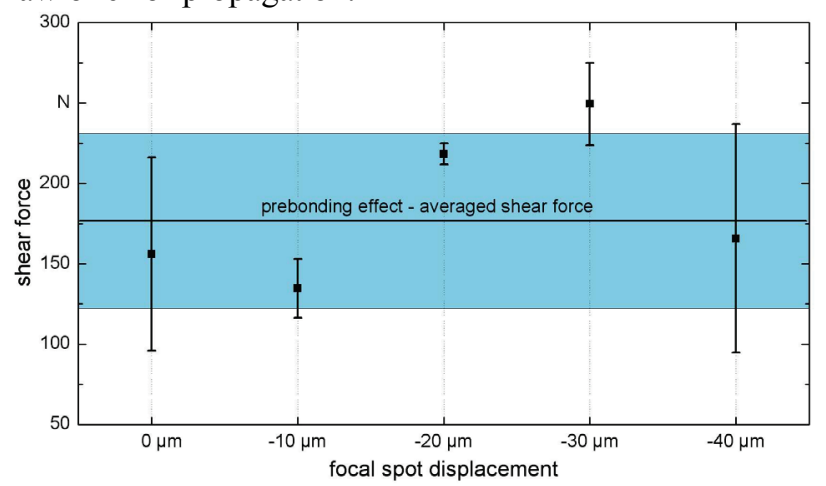

Fig. 3 Averaged shear forces plotted versus the focal displacement. The horizontal line shows the average shear force of the prebonded samples, the blue area depicts the extents of its standard deviation.

The results in table 1 suggest that high shear strengths of the welding seams are probable. However, due to the very high measurement uncertainty which in the case of $20 \mu \mathrm{m}$ focal displacement even exceeds the determined average value no conclusive result for the strength can be given. Only a tendency of higher shear forces in the range of $-20 \mu \mathrm{m}$ and $-30 \mu \mathrm{m}$ can be assumed from this type of experiment.

Table 1 Measurements used for shear strength calculation of optically contacted samples without ethanol immersion.

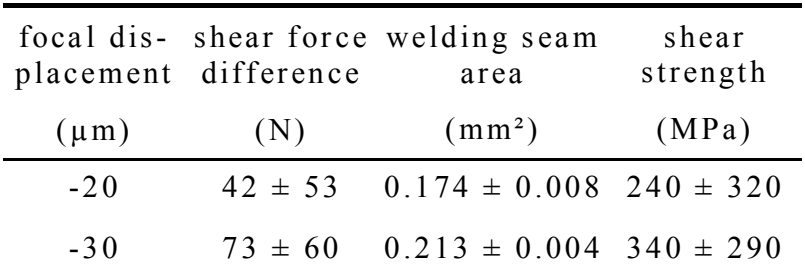

The shear force measurements of welded samples immersed in ethanol are given in fig. 4. However, fig. 4 doesn't show the results for all welded samples. The reasons are the ethanol did not permeate some of the samples sufficiently resulting in shear forces of about $150-200 \mathrm{~N}$ that are comparable to fig. 2. Furthermore, the edge quality of some samples was not sufficiently high resulting in the damage of the glass plate during the shear test, while the optical contact or the welding seam were not broken. Therefore the measurements of these samples were taken out of consideration.

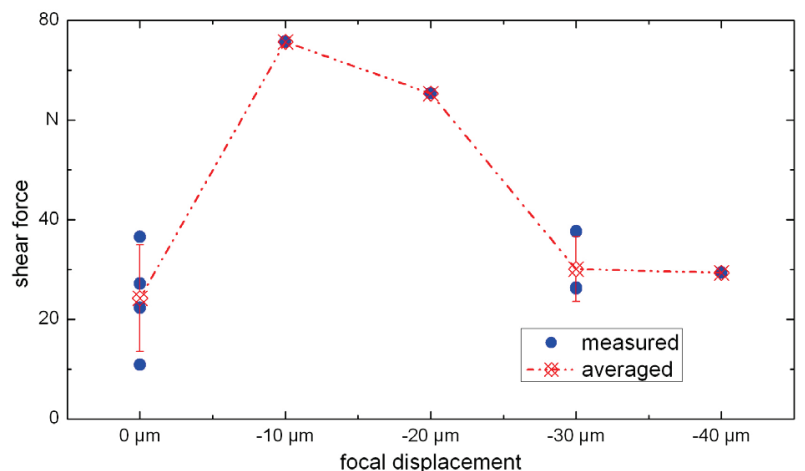

Fig. 4 Shear forces measured in dependence of the focal displacement for welded samples immersed prior to the shear test in ethanol.

It is evident that in fig. 4 the measured shear forces are much lower than in fig. 2. Also in this case the measurements suggest a dependency of the measured shear force on the focal displacement. The shear strength resulting from the measurements in fig. 4 is shown in fig. 5 along with the measured welding seam area. For focal displacements where only a single sample could be evaluated no measurement uncertainties are given.

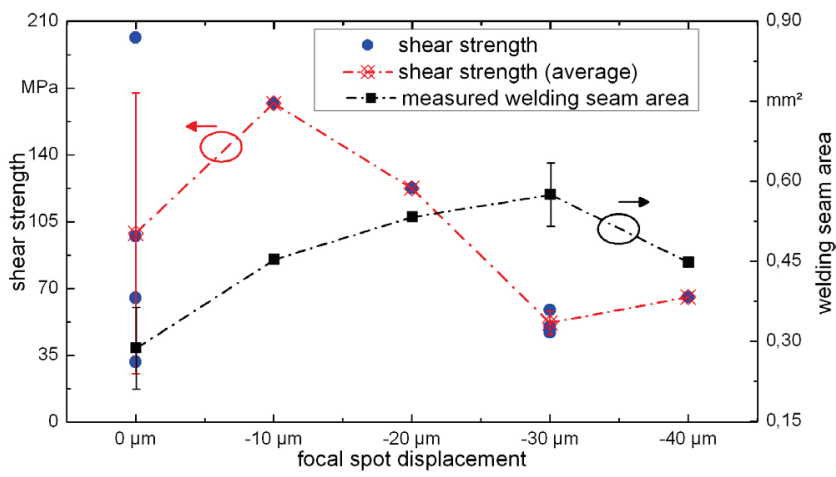

Fig. 5 Shear strength and welding seam areas for samples immersed in ethanol prior to the shear test. 


\section{Discussion}

Fig. 3 and fig. 4 show that the shear force tends to reach its maximum at focal displacements between $-10 \mu \mathrm{m}$ and $-30 \mu \mathrm{m}$. However, only for $-30 \mu \mathrm{m}$ displacement in both experiments a shear force increase was measured for more than one sample.

In the first experiment conducted with prebonded glass samples the measurement precision is compromised due to the fact that the contribution of the optical contact to the overall shear force is larger than the actual shear force of the welding seam. Therefore, although this experiment is fairly straightforward it is not suitable for exact measurements, especially if there are no large quantities of samples available that could be used for reliable statistical analysis.

In the second experiment the influence of prebonded area on the shear force was avoided by immersing the samples in ethanol prior to the shear test. The uncertainty of the measurements in fig. 5 is caused mainly by the lack of samples that could provide statistical support for the measured values since some the samples were either not permeated thoroughly by ethanol or the quality of the glass edge was not high enough to withstand the shear test.

The average shear strengths determined in fig. 5 lie at $50 \mathrm{MPa}$ or higher. The values lie close to the basic shear strength of glass which is in the range of $100-200 \mathrm{MPa}$ [15]. Interestingly the shear strength in fig. 5 does not follow the run of the measured force in fig. 4 . This behavior suggests that probably mechanical tensions are present inside the welding seam that reduce the strength of the seam.

Nonetheless, the high strength across the different displacements shows that the glass welding process is also tolerant to focal displacement other than $-20 \mu \mathrm{m}$, which means that a stable welding process can be easily realized.

A possible cause for errors during both experiments can be found in the surface parallelism of the glass plates. Even though the glass plate roughness was sufficiently low to establish optical contact between the plates thickness deviations of up to $4 \mu \mathrm{m}$ were measured across a single glass plate. This may lead to an error in focal positioning accuracy across the sample even if the upper surface of the sample is horizontal. This error is augmented by the refractive index of glass. This means that in the worst case an experiment done at a certain focal displacement could effectively act as a focal displacement set off geometrically by $10 \mu \mathrm{m}$. However, from the area of the welding seam depicted in fig. 5 can be assumed that this kind of error can probably be neglected since the run of the welding seam seems to follow the typical tear drop shaped cross section of a welding seam [11].

\section{Conclusion and outlook}

As long as the glass samples are joined without defects during ultra-fast-laser welding the achievable shear strengths lie close to the basic strength of the glass material. However, since the welding area depends on the focal displacement with respect to the joining surface higher shear forces are to be expected for broader welding seams.

Indeed, the presented experiments show such behavior, where the maximum shear forces are reached at focal displacements approximately around $-20 \mu \mathrm{m}$. Nonetheless the observed dependency of the shear force on displacement is not highly pronounced. This means that the requirements on the accuracy of the focal position are not stringent and can be chosen in a range of $0 \mu \mathrm{m}$ to $-40 \mu \mathrm{m}$. This is valid for as long as the welding parameter such as average laser power or feed speed are the same as in this experiment. It is to be expected that for lower average laser power or higher feed speeds the accuracy requirements become more stringent as the welding seam height decreases under such conditions. Such conditions might become necessary if either higher welding throughput must be realized or if the available laser power is an issue.

However, the presented experiments make clear that the approach to measure the shear force of the welding seam must be further improved to increase the precision and reliability of the results. The approach which will be used in subsequent experiments will be to open the optical contact after welding by inserting a wedge into the sample from both sides of the welding seam as in a crack opening test [8]. The wedges will be pushed as far as possible towards the welding seam so that only a minimal portion of optical contact remains around the welding seam. Prior to shear test the plates will be immersed in an ethanol solution to further decrease the friction of the optically contacted plates. This way the influence of the optical contact can be reduced in principle completely.

\section{Acknowledgements}

This work was partially supported by Erlangen Graduate School in Advanced Optical Technologies, FriedrichAlexander University Erlangen-Nürnberg.

\section{References}

[1] H. Naumann, and G. Schröder: „Bauelemente der Optik“ (Optical construction elements) (Publisher: Hanser Verlag, Munich, 1992). (in German).

[2] H. Banse: "Laserstrahllöten - Technologie zum Aufbau optischer Systeme" (Laser beam soldering - technology for the setup of optical systems), Ph.D-thesis, University Jena, Germany, 2005. (in German)

[3] V. Greco, F. Marchesini, and G. Molesini, "Optical contact and van der Waals interactions: the role of the surface topography in determining the bonding strength of thick glass plates," J. Opt. A: Pure Appl. Opt., 3, (2001), 85.

[4] S. Hecht-Mijic, A. Harnisch, D. Huelsenberg, S. Schundau, J. Pfeifer and T. Schroeter: „Thermisches Bonden von Bauteilen aus mikrostrukturiertem Glas" (Thermal bonding of elements made of microstructured glass), Mat.-wiss. u. Werkstofftech., 34, (2003), 645. (in German)

[5] H. Maruo, I. Miyamoto, and Y. Arata: "CO2 laser welding of ceramics," 1st International Laser Processing Conference (Laser Institute of America, 1981).

[6] F. Grünwald: „Fertigungsverfahren in der Gerätetechnik" (manufacture processes in device engineering) (Publisher: Carl Hanser Verlag, Munich, Vienna, 1985). (in German)

[7] K. Cvecek, I. Miyamoto, J. Strauss, M. Wolf, T. Frick, and M. Schmidt, "Sample preparation method for glass welding by ultrashort laser pulses yields higher seam strength," Appl. Opt. 50, 1941-1944 (2011). 
[8] Stephan Roth, Kristian Cvecek, Isamu Miyamoto and Michael Schmidt, "Glass welding technology using ultra short laser pulses", Proc. SPIE 7920, 792006 (2011).

[9] E. Sharon and J. Fineberg, "The dynamics of fast fracture," Adv. Eng. Mater. 1, (1999), 119.

[10]K. Cvecek, I. Miyamoto, I. Alexeev and M. Schmidt: "Defect formation in glass welding by means of ultra short laser pulses", Proc. LANE 2010, Erlangen, Germany, Physics Procedia, Volume 5, (2010) p. 495.

[11]I. Miyamoto, K. Cvecek and M. Schmidt: "Evaluation of nonlinear absorptivity in internal modification of bulk glass by ultrashort laser pulses," Opt. Exp., 19, (2011), 10714.

[12] http://www.schott.com/special_applications/english/do wnload/d263te.pdf.
[13] G. Vazquez, E. Alvarez and J. M. Navaza: "Surface Tension of Alcohol + Water from 20 to $50{ }^{\circ} \mathrm{C}$," J. Chem. Eng. Data, 40, (1995), 611.

[14] R. M. Pires, H. F. Costa, A. G. M. Ferreira and I. M. A. Fonseca: "Viscosity and Density of Water + Ethyl Acetate + Ethanol Mixtures at 298.15 and $318.15 \mathrm{~K}$ and Atmosphere Pressure," J. Chem. Eng. Data, 52, (2007), 1240 .

[15] G. Haselhorst, Corporate Machinery and Production Technology, Process and Technology Development, Schott AG (manufacturer of D263 glass), Hattenbergstrasse 10, 55122 Mainz, Germany (personal communication, 2010).

(Received: June 29, 2011, Accepted: January 4, 2012) 